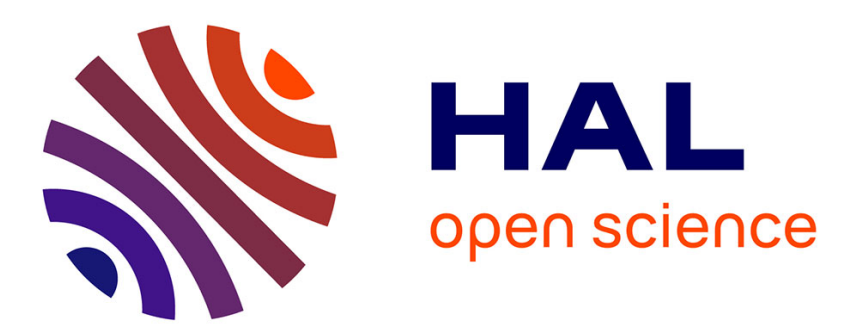

\title{
On the Stokes phenomenon of a family of multi-perturbed level-one meromorphic linear differential systems \\ Pascal Remy
}

\section{- To cite this version:}

Pascal Remy. On the Stokes phenomenon of a family of multi-perturbed level-one meromorphic linear differential systems. Journal of Dynamical and Control Systems, 2013, 19 (4), pp.575-592. 10.1007/s10883-013-9196-1 . hal-00707031v2

\section{HAL Id: hal-00707031 \\ https://hal.science/hal-00707031v2}

Submitted on 24 Oct 2014

HAL is a multi-disciplinary open access archive for the deposit and dissemination of scientific research documents, whether they are published or not. The documents may come from teaching and research institutions in France or abroad, or from public or private research centers.
L'archive ouverte pluridisciplinaire HAL, est destinée au dépôt et à la diffusion de documents scientifiques de niveau recherche, publiés ou non, émanant des établissements d'enseignement et de recherche français ou étrangers, des laboratoires publics ou privés. 


\title{
On the Stokes phenomenon of a family of multi-perturbed level-one meromorphic linear differential systems
}

\author{
P. Remy \\ 6 rue Chantal Mauduit \\ F-78 420 Carrières-sur-Seine \\ email: pascal.remy07@orange.fr
}

\begin{abstract}
Given a level-one meromorphic linear differential system, we investigate the behavior of its Stokes-Ramis matrices under the action of a regular holomorphic perturbation. In particular, we prove that the Stokes-Ramis matrices of the given system can be expressed as limits of convenient product of the perturbed ones. Our approach is based on Écalle's method by regular perturbation and majorant series. No assumption of genericity is made.
\end{abstract}

Keywords. Linear differential system, regular perturbation, holomorphic perturbation, Stokes phenomenon, summability

AMS subject classification. 34M03, 34M30, 34M40

\section{Introduction}

All along the article, we are given a linear differential system (in short, a differential system or a system)

$$
x^{2} \frac{d Y}{d x}=A(x) Y \quad, A(x) \in M_{n}(\mathbb{C}\{x\}), A(0) \neq 0
$$

of dimension $n \geq 2$ with meromorphic coefficients of order 2 at the origin $0 \in \mathbb{C}$. Under the assumption of "single level equal to 1 ", system $(A)$ admits a formal fundamental solution $\widetilde{Y}(x)=\widetilde{F}(x) x^{L} e^{Q(1 / x)}$ where 
- $\widetilde{F}(x) \in M_{n}\left(\mathbb{C}[[x]]\left[x^{-1}\right]\right)$ is an invertible formal meromorphic matrix,

- $L=\bigoplus_{j=1}^{J}\left(\lambda_{j} I_{n_{j}}+J_{n_{j}}\right)$ where $J$ is an integer $\geq 2, I_{n_{j}}$ is the identity matrix of size $n_{j}$ and where

$$
J_{n_{j}}=\left\{\begin{array}{cccc}
0 & & & \\
{\left[\begin{array}{cccc}
0 & 1 & \cdots & 0 \\
\vdots & \ddots & \ddots & \vdots \\
\vdots & & \ddots & 1 \\
0 & \cdots & \cdots & 0
\end{array}\right]} & \text { if } n_{j} \geq 2
\end{array}\right.
$$

is an irreductible Jordan block of size $n_{j}$,

- $Q\left(\frac{1}{x}\right)=\bigoplus_{j=1}^{J}\left(-\frac{a_{j}}{x}\right) I_{n_{j}}$ where the $a_{j} \in \mathbb{C}$ are not equal to a same $a$.

Furthermore, to simplify calculations below, we suppose that the following normalizations of $\widetilde{Y}(x)$ hold:

$(N 1) \widetilde{F}(x) \in M_{n}(\mathbb{C}[[x]])$ is a formal power series in $x$ satisfying $\widetilde{F}(0)=I_{n}$,

(N2) the eigenvalues $\lambda_{j}$ of $L$ satisfy $0 \leq \operatorname{Re}\left(\lambda_{j}\right)<1$ for all $j=1, \ldots, J$,

(N3) $a_{1}=\lambda_{1}=0$.

Recall that such conditions can always be fulfilled by means of a jauge transformation of the form $Y \longmapsto T(x) x^{-\lambda_{1}} e^{a_{1} / x} Y$ where $T(x)$ has explicit computable polynomial entries in $x$ and $1 / x$. Moreover, such a gauge transformation does not affect the Stokes phenomenon of system $(A)$.

Conditions $(N 1)$ and $(N 2)$ guarantee the unicity of $\widetilde{F}(x)$ as formal series solution of the homological system associated with system $(A)$ (cf. [1]). Condition (N3) is for notational convenience.

Observe that normalizations above implies that the matrix $A(x)$ of system (A) reads as

$$
A(x)=\bigoplus_{j=1}^{J}\left(a_{j} I_{n_{j}}+x L_{j}\right)+B(x)
$$


with $L_{j}:=\lambda_{j} I_{n_{j}}+J_{n_{j}}$ the $j$-th Jordan block of $L$ and $B(x)$ analytic at the origin $0 \in \mathbb{C}$; moreover, the assumption "system $(A)$ has the unique level one" is equivalent to the condition

$$
\text { there exists } j \in\{1, \ldots, J\} \text { such that } a_{j} \neq 0
$$

Observe also that, all over the article, no restrictive assumption is made except the assumption that the given system $(A)$ has the unique level one. In particular, we never assume that the formal monodromy $L$ is diagonal nor the Stokes values $a_{j}$ are distinct.

The Stokes phenomenon of system $(A)$ stems from the fact that the sums of $\widetilde{F}(x)$ on each side of a same singular direction (or anti-Stokes direction) of system $(A)$ are not analytic continuations from each other in general; this defect of analyticity is quantified by the Stokes-Ramis matrices (definition $1)$.

The aim of this paper is to study the behavior of these matrices under the action of a holomorphic perturbation acting on the Stokes values $a_{j} \neq 0$. In particular, we prove that they are limits of convenient products of the Stokes-Ramis matrices of the perturbed systems.

The organization of the paper is as follows: in section 1, we recall for the convenience of the reader some definitions about the summation theory. In section 2 , we introduce a regular perturbation of system $(A)$ of the form

$$
x^{2} \frac{d Y}{d x}=A^{\varepsilon}(x) Y
$$

with

$$
A^{\varepsilon}(x)=\bigoplus_{j=1}^{J}\left(a_{j}^{\varepsilon} I_{n_{j}}+x L_{j}\right)+B(x) \quad, A^{\mathbf{1}}(x)=A(x)
$$

where $\varepsilon$ is a holomorphic multi-parameter acting on the Stokes values $a_{j}$ 's (compare with (0.1)) and lying in a polydisc centered at the unit $\mathbf{1}:=(1, \ldots, 1)$ of the $\mathbb{C}$-vector space $\mathbb{C}^{p}$ for a convenient $p \geq 1$. Doing so, the perturbation acts on the anti-Stokes directions of initial system $(A)$ and changes them into anti-Stokes directions of systems $\left(A^{\varepsilon}\right)$. Then, we first describe precisely the geometry of the perturbed ones and select some Stokes matrices ${ }^{1}$ which are

\footnotetext{
${ }^{1}$ In the whole paper, we call Stokes matrices all the matrices providing the transition between any two asymptotic solutions whose domains of definition overlap. The name "Stokes-Ramis matrix" is reserved, according to the custom initiated by J.-P. Ramis ([4]) in the spirit of Stokes' work, to the matrices providing the transition between the sums on each side of a same anti-Stokes direction. Thereby, a Stokes-Ramis matrix is a Stokes Ramis, but the converse is false in general. Nevertheless, all Stokes matrices are finite products of convenient Stokes-Ramis matrices.
} 
proved to depend holomorphically on the parameter $\varepsilon$ and to converge to the Stokes-Ramis matrices of initial system $(A)$ when $\varepsilon$ goes to $\mathbf{1}$ (theorem 1 ). The proof of this result, which is essentially based on an adequate variant of the proof of summable-resurgence theorem following Écalle's method by regular perturbation and majorant series displayed by M. Loday-Richaud and the author in [3], is developed in section 3.

\section{Some definitions and notations}

\subsection{Stokes values and anti-Stokes directions}

Split the matrix $\widetilde{F}(x)=\left[\begin{array}{lll}\widetilde{F}^{\bullet ; 1}(x) & \cdots & \widetilde{F}^{\bullet ;}(x)\end{array}\right]$ into $J$ column-blocks fitting the Jordan structure of $L$ (hence, the size of $\widetilde{F}^{\bullet ; k}(x)$ is $n \times n_{k}$ for all $k$ ).

Let $\Omega:=\left\{a_{j}, j=1, \ldots, J\right\}$ denote the set of Stokes values of system $(A)$. The directions determined by the elements of $\Omega^{*}:=\Omega \backslash\{0\}$ from 0 are called anti-Stokes directions associated with $\widetilde{F}^{\bullet} ; 1(x)$.

The anti-Stokes directions associated with the $k$-th column-block $\widetilde{F}^{\bullet ; k}(x)$ of $\widetilde{F}(x)$ are given by the nonzero elements of $\Omega-a_{k}$ (to normalize the $k$ th column-block, one has to multiply by $\left.e^{a_{k} / x}\right)$; the anti-Stokes directions of system $(A)$, i.e., associated with the full matrix $\widetilde{F}(x)$, are given by the nonzero elements of $\Omega:=\left\{a_{j}-a_{k}, j, k=1, \ldots, J\right\}$. Recall that the elements of $\boldsymbol{\Omega}$ are the Stokes values of the homological system associated with system $(A)$.

\subsection{Summation, Stokes phenomenon and Stokes-Ramis matrices}

- Given a non anti-Stokes direction $\theta \in \mathbb{R} / 2 \pi \mathbb{Z}$ of system $(A)$ and a choice of an argument of $\theta$, say its principal determination $\left.\left.\theta^{\star} \in\right]-2 \pi, 0\right]^{2}$, we consider the sum of $\widetilde{Y}$ in the direction $\theta$ given by

$$
Y_{\theta}(x)=s_{1 ; \theta}(\widetilde{F})(x) Y_{0, \theta^{\star}}(x)
$$

where $s_{1 ; \theta}(\widetilde{F})(x)$ is the uniquely determined 1-sum (or Borel-Laplace sum) of $\widetilde{F}(x)$ at $\theta$ and where $Y_{0, \theta^{\star}}(x)$ is the actual analytic function $Y_{0, \theta^{\star}}(x):=$ $x^{L} e^{Q(1 / x)}$ defined by the choice $\arg (x)$ close to $\theta^{\star}$ (denoted below $\left.\arg (x) \simeq \theta^{\star}\right)$.

\footnotetext{
${ }^{2}$ Any choice is convenient. However, to be compatible, on the Riemann sphere, with the usual choice $0 \leq \arg (z=1 / x)<2 \pi$ of the principal determination at infinity, we suggest to choose $-2 \pi<\arg (x) \leq 0$ as principal determination about 0 .
} 
Recall that $s_{1 ; \theta}(\widetilde{F})$ is an analytic function defined and 1-Gevrey asymptotic to $\widetilde{F}$ on a germ of sector bisected by $\theta$ and opening larger than $\pi$.

Recall also that $s_{1 ; \theta}(\widetilde{F})(x)$ is given by the Borel-Laplace integral

$$
\int_{0}^{\infty e^{i \theta}} \widehat{F}(\xi) e^{-\xi / x} d \xi
$$

where $\widehat{F}(\xi)$ denotes the Borel transform of $\widetilde{F}(x)$.

- When $\theta \in \mathbb{R} / 2 \pi \mathbb{Z}$ is an anti-Stokes direction of system $(A)$, we consider the two lateral sums $s_{1 ; \theta^{-}}(\widetilde{F})$ and $s_{1 ; \theta^{+}}(\widetilde{F})$ respectively obtained as analytic continuations of $s_{1 ; \theta-\eta}(\widetilde{F})$ and $s_{1 ; \theta+\eta}(\widetilde{F})$ to a germ of half-plane bisected by $\theta$. Note that such analytic continuations exist without ambiguity when $\eta>0$ is small enough.

We denote by $Y_{\theta^{-}}$and $Y_{\theta^{+}}$the sums of $\widetilde{Y}$ respectively defined for $\arg (x) \simeq$ $\theta^{\star}$ by $Y_{\theta^{-}}(x):=s_{1 ; \theta^{-}}(\widetilde{F})(x) Y_{0, \theta^{\star}}(x)$ and $Y_{\theta^{+}}(x):=s_{1 ; \theta^{+}}(\widetilde{F})(x) Y_{0, \theta^{\star}}(x)$.

The Stokes phenomenon of system $(A)$ stems from the fact that the sums $s_{1 ; \theta^{-}}(\widetilde{F})$ and $s_{1 ; \theta^{+}}(\widetilde{F})$ of $\widetilde{F}$ are not analytic continuations from each other in general. This defect of analyticity is quantified by the collection of StokesRamis automorphisms

$$
S t_{\theta^{\star}}: Y_{\theta^{+}} \longmapsto Y_{\theta^{-}}
$$

for all the anti-Stokes directions $\theta \in \mathbb{R} / 2 \pi \mathbb{Z}$ of system $(A)$.

The Stokes-Ramis matrices are defined as matrix representations of the $S t_{\theta^{\star}}$ 's in $G L_{n}(\mathbb{C})$.

\section{Definition 1.1 (Stokes-Ramis matrices)}

One calls Stokes-Ramis matrix associated with $\widetilde{Y}$ in the direction $\theta$ the matrix of $S t_{\theta^{\star}}$ in the basis $Y_{\theta^{+}}$. We still denote it $S t_{\theta^{\star}}$.

Note that the matrix $S t_{\theta^{\star}}$ is uniquely determined by the relation

$$
Y_{\theta^{-}}(x)=Y_{\theta^{+}}(x) S t_{\theta^{\star}} \quad \text { for } \arg (x) \simeq \theta^{\star}
$$

\section{Setting the problem}

We denote below by

- $D(\alpha, \rho):=\{x \in \mathbb{C} ;|x-\alpha|<\rho\}$ the open disc in $\mathbb{C}$ with midpoint $\alpha \in \mathbb{C}$ and radius $\rho>0$, 
- $\bar{D}(\alpha, \rho):=\{x \in \mathbb{C} ;|x-\alpha| \leq \rho\}$ the closed disc in $\mathbb{C}$ with midpoint $\alpha \in \mathbb{C}$ and radius $\rho>0$,

- $\Sigma_{\theta, \eta}:=\left\{x \in \mathbb{C}^{*} ;|\arg (x)-\theta|<\eta / 2\right\}$ the open sector in $\mathbb{C}^{*}$ with vertex 0 , bisected by $\theta \in \mathbb{R} / 2 \pi \mathbb{Z}$ and opening $\eta>0$,

- $\bar{\Sigma}_{\theta, \eta}:=\left\{x \in \mathbb{C}^{*} ;|\arg (x)-\theta| \leq \eta / 2\right\}$ the closure of $\Sigma_{\theta, \eta}$ in $\mathbb{C}^{*}$ (hence, we refer $\bar{\Sigma}_{\theta, \eta}$ as a closed sector).

\subsection{A multi-perturbed system}

In addition to notations above, we denote in this section by $\omega_{1}, \ldots, \omega_{p}$ with $p \geq 1$ the nonzero Stokes values of system $(A)$. Hence,

$$
\Omega=\left\{\omega_{0}:=0\right\} \cup\left\{\omega_{k}, k=1, \ldots, p\right\}
$$

and

$$
\boldsymbol{\Omega}=\left\{\omega_{0}:=0\right\} \cup\left\{\omega_{k}-\omega_{\ell}, k, \ell=0, \ldots, p \text { and } k \neq \ell\right\} .
$$

Note that $\omega_{k}-\omega_{\ell} \neq 0$ for all $k \neq \ell$.

According to normalizations $(N 1)-(N 3)$ of $\tilde{Y}(x)$ (cf. page 2), the matrix $A(x)$ of system $(A)$ reads

$$
A(x)=\bigoplus_{j=1}^{J}\left(a_{j} I_{n_{j}}+x L_{j}\right)+B(x)
$$

where $a_{j}=\omega_{k}$ for a certain $k \in\{0, \ldots, p\}, L_{j}:=\lambda_{j} I_{n_{j}}+J_{n_{j}}$ denotes the $j$-th Jordan block of the matrix $L$ of exponents of formal monodromy and where $B(x)$ is analytic at the origin $0 \in \mathbb{C}$.

From now, we are given

(1) a parameter $\varepsilon:=\left(\varepsilon_{1}, \ldots, \varepsilon_{p}\right)$ in a polydisc $\mathcal{D}_{p}:=D\left(1, \rho_{1}\right) \times \ldots \times D\left(1, \rho_{p}\right)$ of $\mathbb{C}^{p}$; conditions on the $\rho_{k}$ 's are precised below,

(2) the regularly multi-perturbed system

$$
x^{2} \frac{d Y}{d x}=A^{\varepsilon}(x) Y
$$

where

$$
A^{\varepsilon}(x)=\bigoplus_{j=1}^{J}\left(a_{j}^{\varepsilon} I_{n_{j}}+x L_{j}\right)+B(x)
$$


with

$$
a_{j}^{\varepsilon}=\left\{\begin{array}{ll}
0 & \text { if } a_{j}=\omega_{0}=0 \\
\omega_{k} \varepsilon_{k} & \text { if } a_{j}=\omega_{k} \text { and } k \in\{1, \ldots, p\}
\end{array} .\right.
$$

Note that, for $\boldsymbol{\varepsilon}=\mathbf{1}:=(1, \ldots, 1)$ the unit of $\mathbb{C}^{p}$, we have $A^{\mathbf{1}} \equiv A$ and systems $\left(A^{\mathbf{1}}\right)$ and $(A)$ coincide. Note also that

$$
\omega_{k} \varepsilon_{k} \in D\left(\omega_{k},\left|\omega_{k}\right| \rho_{k}\right) \quad \text { for all } k=1, \ldots, p .
$$

Hence, under the two conditions

(C1) $0 \notin \bar{D}\left(\omega_{k},\left|\omega_{k}\right| \rho_{k}\right)$ for all $k=1, \ldots, p$,

$(C 2) \bar{D}\left(\omega_{k},\left|\omega_{k}\right| \rho_{k}\right) \cap \bar{D}\left(\omega_{\ell},\left|\omega_{\ell}\right| \rho_{\ell}\right)=\emptyset$ for all $k, \ell=1, \ldots, p, k \neq \ell$,

which are always satisfied as soon as the $\rho_{k}$ 's are small enough, system $\left(A^{\varepsilon}\right)$ has, for all $\varepsilon \in \mathcal{D}_{p}$, the unique level 1 and has for formal fundamental solution the matrix $\widetilde{Y}^{\varepsilon}(x)=\widetilde{F}^{\varepsilon}(x) x^{L} e^{Q^{\varepsilon}(1 / x)}$ where

- $\widetilde{F}^{\varepsilon}(x) \in M_{n}(\mathbb{C}[[x]])$ is a power series in $x$ verifying $\widetilde{F}^{\varepsilon}(0)=I_{n}$,

- $L$ is the matrix of exponents of formal monodromy of system $(A)$,

- $Q^{\varepsilon}(1 / x)=\bigoplus_{j=1}^{J}\left(-a_{j}^{\varepsilon} / x\right) I_{n_{j}}$.

Note that, like systems $\left(A^{\varepsilon}\right)$ and $(A)$, the two formal fundamental solutions $\widetilde{Y}^{\varepsilon}(x)$ and $\widetilde{Y}(x)$ coincide for $\varepsilon=1$. Note also that $\widetilde{Y}^{\varepsilon}(x)$ has same normalizations as $\widetilde{Y}(x)$ for all $\varepsilon \in \mathcal{D}_{p}$.

We shall now give some basic geometric properties of the Stokes values and the anti-Stokes directions of systems $\left(A^{\varepsilon}\right)$.

\subsection{Action of the perturbation on the Stokes values, singular discs}

For any $\varepsilon \in \mathcal{D}_{p}$, we denote by

- $\Omega^{\varepsilon}$ the set of Stokes values of system $\left(A^{\varepsilon}\right)$,

- $\Omega^{\varepsilon}$ the set of Stokes values of the homological system associated with $\left(A^{\varepsilon}\right)$.

By construction, $\Omega^{\varepsilon}$ (resp. $\Omega^{\varepsilon}$ ) is deduced from $\Omega$ (resp. $\Omega$ ) by replacing the Stokes values $\omega_{k}, k=1, \ldots, p$ (resp. $\omega_{k}-\omega_{\ell}, k, \ell=0, \ldots, p$ and $k \neq \ell$ ) by the perturbed Stokes values $\omega_{k} \varepsilon_{k}$ (resp. $\omega_{k} \varepsilon_{k}-\omega_{\ell} \varepsilon_{\ell}$ ). Hence, 
- $\Omega^{\varepsilon}=\{0\} \cup\left\{\omega_{k} \varepsilon_{k}, k=1, \ldots, p\right\}$,

- $\Omega^{\varepsilon}=\{0\} \cup\left\{\omega_{k} \varepsilon_{k}-\omega_{\ell} \varepsilon_{\ell}, k, \ell=0, \ldots, p\right.$ and $\left.k \neq \ell\right\}$; we set $\varepsilon_{0}:=1$.

Note that, due to conditions $(C 1)$ and $(C 2), \omega_{k} \varepsilon_{k}-\omega_{\ell} \varepsilon_{\ell} \neq 0$ for all $k \neq \ell$.

We denote also by

- $\Omega\left(\mathcal{D}_{p}\right):=\bigcup_{\varepsilon \in \mathcal{D}_{p}} \Omega^{\varepsilon}$ the set of all the Stokes values of all systems $\left(A^{\varepsilon}\right)$ when $\varepsilon$ runs in $\mathcal{D}_{p}$,

- $\Omega\left(\mathcal{D}_{p}\right):=\bigcup_{\varepsilon \in \mathcal{D}_{p}} \Omega^{\varepsilon}$ the set of all the Stokes values of all the homological systems associated with all systems $\left(A^{\varepsilon}\right)$ when $\varepsilon$ runs in $\mathcal{D}_{p}$.

The sets $\Omega\left(\mathcal{D}_{p}\right)$ and $\Omega\left(\mathcal{D}_{p}\right)$ are the respective "images" of $\Omega$ and $\Omega$ under the action of the perturbation in $\varepsilon$. More precisely,

$$
\begin{aligned}
& \text { - } \Omega\left(\mathcal{D}_{p}\right)=\{0\} \cup\left(\bigcup_{k=1}^{p} D\left(\omega_{k},\left|\omega_{k}\right| \rho_{k}\right)\right) \\
& \text { - } \Omega\left(\mathcal{D}_{p}\right)=\{0\} \cup\left(\bigcup_{\substack{k, \ell=0 \\
k \neq \ell}}^{p} D\left(\omega_{k}-\omega_{\ell},\left|\omega_{k}\right| \rho_{k}+\left|\omega_{\ell}\right| \rho_{\ell}\right)\right) \text {; we set } \rho_{0}:=1 .
\end{aligned}
$$

Note that $\Omega \subset \Omega$ implies $\Omega\left(\mathcal{D}_{p}\right) \subset \Omega\left(\mathcal{D}_{p}\right)$. Note also that, unlike to $\Omega\left(\mathcal{D}_{p}\right)$, some discs of $\Omega\left(\mathcal{D}_{p}\right)$ may overlap.

By construction, the disc $D_{\omega_{k}-\omega_{\ell}}:=D\left(\omega_{k}-\omega_{\ell},\left|\omega_{k}\right| \rho_{k}+\left|\omega_{\ell}\right| \rho_{\ell}\right)$ is formed, for any $k \neq \ell$, by all the points $\omega_{k} \varepsilon_{k}-\omega_{\ell} \varepsilon_{\ell} \in \Omega\left(\mathcal{D}_{p}\right)$ issuing from the nonzero Stokes value $\omega_{k}-\omega_{\ell} \in \Omega$ under the action of the perturbation. This brings us to the following definition:

\section{Definition 2.1 (Singular disc of $\Omega\left(\mathcal{D}_{p}\right)$ )}

Let $\omega:=\omega_{k}-\omega_{\ell}$ be a nonzero Stokes value of $\Omega$. Then, the disc $D_{\omega}:=D_{\omega_{k}-\omega_{\ell}}$ is called singular disc of $\Omega\left(\mathcal{D}_{p}\right)$ associated with $\omega$.

Remark 2.2 Observe that, due to conditions $(C 1)$ and $(C 2)$, none of the closed singular disc $\bar{D}_{\omega}\left(=\right.$ the closure of $D_{\omega}$ in $\left.\mathbb{C}\right)$ contains 0 .

Remark 2.3 Relations above between initial Stokes values and perturbed Stokes values have a translation in terms of anti-Stokes directions: let $\theta \in$ $\mathbb{R} / 2 \pi \mathbb{Z}$ be an anti-Stokes direction of initial system $(A)$; then, its "image" by the perturbation is the set of all the anti-Stokes directions of all systems $\left(A^{\varepsilon}\right)$ given by all the points of all the singular discs of $\Omega\left(\mathcal{D}_{p}\right)$ centered on $\theta$.

Remark 2 will be precised in lemma 1 below. 


\subsection{Action of the perturbation on the anti-Stokes dir- ections, singular sectors}

In addition to previous notations, we also denote by

- $\Theta$ the set of anti-Stokes directions of initial system $(A)$,

- $\boldsymbol{\Omega}_{\theta}$ the set of nonzero Stokes values of $\Omega$ with argument $\theta \in \mathbb{R} / 2 \pi \mathbb{Z}$.

Obviously, $\theta \in \Theta$ if and only if $\Omega_{\theta} \neq \emptyset$. For any $\theta \in \Theta$, we consider

- $\boldsymbol{\Omega}_{\theta}\left(\mathcal{D}_{p}\right):=\bigcup_{\omega \in \boldsymbol{\Omega}_{\theta}} D_{\omega}$ the set of all the singular discs $D_{\omega}$ of $\boldsymbol{\Omega}\left(\mathcal{D}_{p}\right)$ associated with all the Stokes values $\omega \in \Omega_{\theta}$, i.e., the set of all the singular discs of $\Omega\left(\mathcal{D}_{p}\right)$ centered on $\theta$.

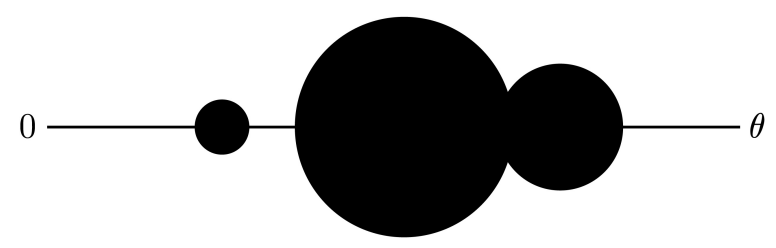

Figure 1 - A set $\Omega_{\theta}\left(\mathcal{D}_{p}\right)$

Since all the discs $D_{\omega}$ with $\omega \in \Omega_{\theta}$ are symmetrical about $\theta$, we also consider

- $\eta(\theta)$ the minimal opening of sectors $\Sigma_{\theta, \eta}$ containing $\boldsymbol{\Omega}_{\theta}\left(\mathcal{D}_{p}\right)$.

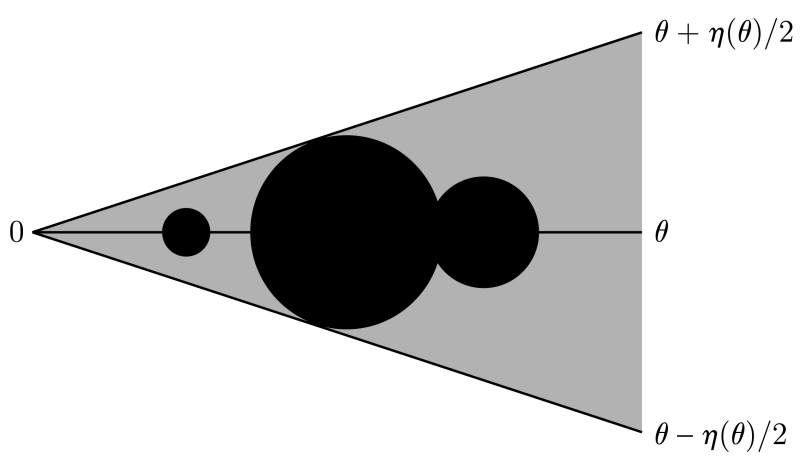

Figure 2 - A sector $\Sigma_{\theta, \eta(\theta)}$ 
By construction, the directions determined by the points of $\Sigma_{\theta, \eta(\theta)}$ are the anti-Stokes directions of systems $\left(A^{\varepsilon}\right)$ determined by all the points of $\boldsymbol{\Omega}_{\theta}\left(\mathcal{D}_{p}\right)$. Thereby, remark 2 leads us to the following lemma.

Lemma 2.4 (Action of the perturbation on $\theta \in \Theta$ )

Let $\theta \in \Theta$ be an anti-Stokes direction of initial system $(A)$.

Then, the "image" of $\theta$ by the perturbation is the set $D \Sigma_{\theta, \eta(\theta)}$ of all the directions determined by the points of $\Sigma_{\theta, \eta(\theta)}$.

Before stating the main result of the article (see theorem 1 below), let us make some remarks about sectors $\Sigma_{\theta, \eta(\theta)}$. First, their openings $\eta(\theta)$ only depend on the radius of the singular discs $D_{\omega}$ associated with $\omega \in \Omega_{\theta}$. In particular, the $\eta(\theta)$ 's tend to 0 when the $\rho_{k}$ 's go to 0 . Second, the size of the $\eta(\theta)$ 's will play a fundamental role in theorem 1 (see section 2.4 below). Henceforth, we suppose that the radius $\rho_{k}$ 's are chosen small enough so that, in addition to conditions $(C 1)$ and $(C 2)$ above, the following conditions would be satisfied:

(C3) $\bar{\Sigma}_{\theta, \eta(\theta)} \cap \bar{\Sigma}_{\theta^{\prime}, \eta\left(\theta^{\prime}\right)}=\emptyset$ for all $\theta, \theta^{\prime} \in \Theta, \theta \neq \theta^{\prime}$,

(C4) for all $\theta \in \Theta, \eta(\theta)<\frac{\pi}{2}$,

(C5) for all $\theta \in \Theta$, the principal determination $\theta^{\star}$ of $\theta$ and the principal determination $(\theta-\eta(\theta) / 2)^{\star}$ of $\theta-\eta(\theta) / 2$ satisfy

$$
-2 \pi<(\theta-\eta(\theta) / 2)^{\star}<\theta^{\star} \leq 0
$$

Remark 2.5 Let $D \bar{\Sigma}_{\theta, \eta(\theta)}$ denote the set of all the directions determined by all the points of the closed sector $\bar{\Sigma}_{\theta, \eta(\theta)}$. Condition (C3) above tells us that $D \bar{\Sigma}_{\theta, \eta(\theta)}$ contains no other anti-Stokes directions of systems $\left(A^{\varepsilon}\right), \varepsilon$ running in $\mathcal{D}_{p}$, except those issuing from $\theta$ under the action of the perturbation. In particular, since systems $(A)$ and $\left(A^{\varepsilon}\right)$ coincide for $\varepsilon=\mathbf{1}$, the set $D \bar{\Sigma}_{\theta, \eta(\theta)}$ just contains the direction $\theta$ as anti-Stokes directions of system $(A)$.

\subsection{Main result}

As before, we indicate by

- $\Theta$ the set of anti-Stokes directions of initial system $(A)$;

- $D \Sigma_{\theta, \eta}$ (resp. $D \bar{\Sigma}_{\theta, \eta}$ ) the set of all the directions determined by all the points of $\Sigma_{\theta, \eta}\left(\operatorname{resp} . \bar{\Sigma}_{\theta, \eta}\right)$ for any $\eta>0$. 
Let $\theta \in \Theta$ and $D \Sigma_{\theta, \eta(\theta)}$ its "image" by the perturbation ( $c f$. lemma 1). Under conditions $(C 3)-(C 5)$ above, there exists $\eta \in] \eta(\theta), \pi / 2[$ such that

1. $\Sigma_{\theta, \eta(\theta)} \varsubsetneqq \Sigma_{\theta, \eta} \varsubsetneqq \Sigma_{\theta, \pi-\eta}$,

2. $\bar{\Sigma}_{\theta, \eta} \cap \bar{\Sigma}_{\theta^{\prime}, \eta\left(\theta^{\prime}\right)}=\emptyset$ for all $\theta^{\prime} \in \Theta, \theta^{\prime} \neq \theta$,

3. the principal determination $(\theta-\eta / 2)^{\star}$ of $\theta-\eta / 2$ satisfies

$$
-2 \pi<(\theta-\eta / 2)^{\star}<(\theta-\eta(\theta) / 2)^{\star}<\theta^{\star} \leq 0
$$

Note that point 1 . results from the choice $\eta$ in $] \eta(\theta), \pi / 2[$ and that points 2. and 3. hold as soon as $\eta$ is close enough to $\eta(\theta)$. Note also that point 2. guarantees that the set $D \bar{\Sigma}_{\theta, \eta}$ contains no other anti-Stokes directions of systems $\left(A^{\varepsilon}\right), \varepsilon$ running in $\mathcal{D}_{p}$, except those of $D \Sigma_{\theta, \eta(\theta)}$.

Let us now fix $\varepsilon \in \mathcal{D}_{p}$ and $\eta$ as above. Then, according to points 1.3., directions $\theta \pm \eta / 2$ are not anti-Stokes directions of system $\left(A^{\varepsilon}\right)$ and the 1-sums $s_{1 ; \theta \pm \eta / 2}\left(\widetilde{F}^{\varepsilon}\right)$ are thus defined and analytic on a same germ of sector $\Sigma_{\theta, \pi-\eta}$. Consequently, the sums

$$
Y_{\theta \pm \eta / 2}^{\varepsilon}(x):=s_{1 ; \theta \pm \eta / 2}\left(\widetilde{F}^{\varepsilon}\right)(x) x^{L} e^{Q^{\varepsilon}(1 / x)}
$$

are related for $\arg (x) \in](\theta-\eta / 2)^{\star},(\theta-\eta(\theta) / 2)^{\star}[$ (see figure 3 below) by the relation

$$
Y_{\theta-\eta / 2}^{\varepsilon}(x)=Y_{\theta+\eta / 2}^{\varepsilon}(x) \mathfrak{S}_{\theta^{\star}}^{\varepsilon}
$$

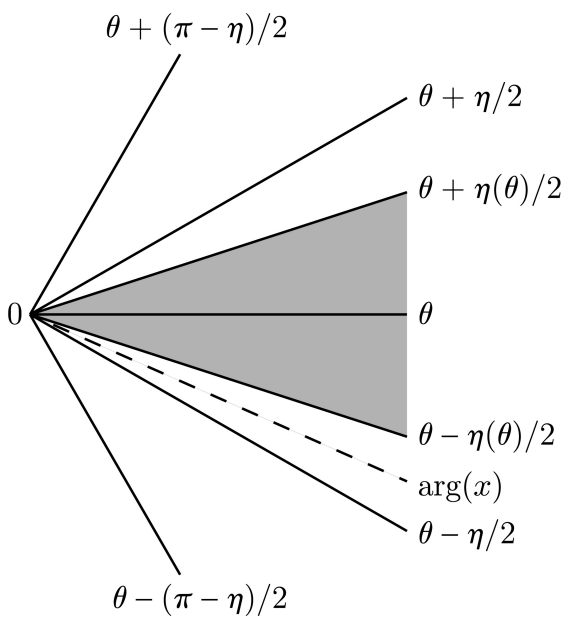

Figure 3 - Sector $\Sigma_{\theta, \eta(\theta)}$ and associated directions 
The matrix $\mathfrak{S}_{\theta^{\star}}^{\varepsilon} \in G L_{n}(\mathbb{C})$ denotes the (perturbed) connection matrix between $Y_{\theta+\eta / 2}^{\varepsilon}$ and $Y_{\theta-\eta / 2}^{\varepsilon}$. It is only determined by identity (2.1) above. Note that remark 3 and point 2 . above imply that $\mathfrak{S}_{\theta^{\star}}^{\varepsilon}$ is actually defined as (finite) product of Stokes-Ramis matrices associated with $\tilde{Y}^{\varepsilon}$ in the anti-Stokes directions of system $\left(A^{\varepsilon}\right)$ contained in $D \Sigma_{\theta, \eta(\theta)}$. Note also that, for $\boldsymbol{\varepsilon}=\mathbf{1}$, we have

$$
Y_{\theta \pm \eta / 2}^{1}(x)=Y_{\theta \pm \eta / 2}(x)=Y_{\theta^{ \pm}}(x) \quad \text { and } \quad \mathfrak{S}_{\theta^{\star}}^{1} \equiv S t_{\theta^{\star}}
$$

We are now able to state the main result of the article:

Theorem 2.6 Let $\theta \in \Theta$ be an anti-Stokes direction of initial system (A). Then,

1. the function $\varepsilon \longmapsto \mathfrak{S}_{\theta^{\star}}^{\varepsilon}$ is holomorphic on $\mathcal{D}_{p}$,

2. the Stokes-Ramis matrix St $t_{\theta^{\star}}$ of initial system $(A)$ is limit of the perturbed Stokes matrices $\mathfrak{S}_{\theta^{\star}}^{\varepsilon}$ :

$$
\lim _{\varepsilon \rightarrow \mathbf{1}} \mathfrak{S}_{\theta^{\star}}^{\varepsilon}=S t_{\theta^{\star}}
$$

Before starting the proof of theorem 1, let us make some remarks. First, it is clear that point 2 . is straightforward from point 1 . Indeed, we have $\mathfrak{S}_{\theta^{\star}}^{1} \equiv$ $S t_{\theta^{\star}}$ by definition of the perturbation (see relations (2.2) above). Second, it seems that identity (2.3) could provide an efficient tool for the effective calculation of Stokes-Ramis matrices of initial system $(A)$. This "question", which is one of our actual directions of research, will be investigated in great detail in [5].

We now turn to the proof of theorem 1 .

\section{Proof of theorem 1}

As mentioned above, we are left to prove the first point of theorem 1 . The central point of this proof is the study of the 1-sums $s_{1 ; \theta \pm \eta / 2}\left(\widetilde{F}^{\varepsilon}\right)(x)$ following the parameter $\varepsilon$. Precisely, we shall show in proposition 2 below that, on one hand, these sums are defined for all $\varepsilon \in \mathcal{D}_{p}$ on a same germ $\Sigma$ of sector

$$
\left\{x \in \mathbb{C}^{*} ;\left(\theta-\frac{\eta}{2}\right)^{\star}<\arg (x)<\left(\theta-\frac{\eta(\theta)}{2}\right)^{\star}\right\}
$$


and, on the other hand, they are holomorphic on $\mathcal{D}_{p}$ for all $x \in \Sigma$. However, before studying the $s_{1 ; \theta \pm \eta / 2}\left(\widetilde{F}^{\varepsilon}\right)(x)^{\text {'s }}$, we shall first investigate the Borel transforms $\widehat{F}^{\varepsilon}(\xi)$ of $\widetilde{F}^{\varepsilon}(x)$ (with respect to $x$ ). Recall indeed that $s_{1 ; \theta \pm \eta / 2}\left(\widetilde{F}^{\varepsilon}\right)(x)$ and $\widehat{F}^{\varepsilon}(\xi)$ are related by the integral formula

$$
\int_{0}^{\infty e^{i(\theta \pm \eta / 2)}} \widehat{F}^{\varepsilon}(\xi) e^{-\xi / x} d \xi
$$

Recall also, for the convenience of the reader, that the formal Borel transformation is an isomorphism from the $\mathbb{C}$-differential algebra $\left(\mathbb{C}[[x]],+, \cdot, x^{2} \frac{d}{d x}\right)$ to the $\mathbb{C}$-differential algebra $(\delta \mathbb{C} \oplus \mathbb{C}[[\xi]],+, *, \xi \cdot)$ that changes ordinary product - into convolution product $*$ and also changes derivation $x^{2} \frac{d}{d x}$ into multiplication by $\xi$. It also changes multiplication by $\frac{1}{x}$ into derivation $\frac{d}{d \xi}$. Moreover, if $g(x) \in \mathbb{C}\{x\}$ is an analytic function at the origin $0 \in \mathbb{C}$, then its formal Borel transform $\widehat{g}(\xi)$ defines an entire function on all $\mathbb{C}$ with exponential growth at infinity.

\subsection{Dependence in $\varepsilon$ and Borel transform}

Recall that $\mathcal{D}_{p}$ denotes the polydisc $D\left(1, \rho_{1}\right) \times \ldots \times D\left(1, \rho_{p}\right)$ in $\mathbb{C}^{p}$ where the radius $\rho_{k}$ 's are chosen so that conditions $(C 1)-(C 5)$ hold. Recall also that, for any nonzero Stokes value $\omega \in \Omega, D_{\omega}$ denotes the singular disc of $\Omega\left(\mathcal{D}_{p}\right)$ associated with $\omega$, i.e., the open disc formed by all the Stokes values of $\Omega\left(\mathcal{D}_{p}\right)$ issuing from $\omega$ under the action of the perturbation.

In this section, we consider a domain $V \subset \mathbb{C}$ defined by the data of an open disc centered at $0 \in \mathbb{C}$ and an open sector in $\mathbb{C}$ with vertex 0 such that

$$
\bar{V} \cap \bar{D}_{\omega}=\emptyset \quad \text { for all } \omega \in \Omega \backslash\{0\}
$$

( $\bar{V}$ and $\bar{D}_{\omega}$ denote respectively the closure of $V$ and $D_{\omega}$ in $\mathbb{C}$ ). Observe that the existence of such a domain $V$ is ensured by conditions $(C 1)-(C 3)$ and 
remark 1.

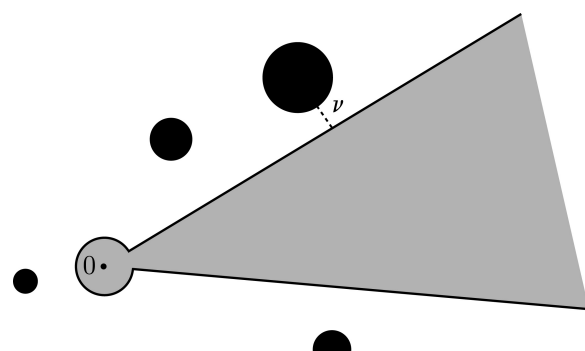

Figure 4 - A domain $V$ and the singular discs $D_{\omega}$ of $\Omega\left(\mathcal{D}_{p}\right)$

Our aim is to prove the following result:

Proposition 3.1 Let $V$ be a domain as above.

Then, the function $(\xi, \varepsilon) \longmapsto \widehat{F}^{\varepsilon}(\xi)$ is holomorphic on $V \times \mathcal{D}_{p}$.

Proposition 1 is proved below by using an adequate variant of the proof of summable-resurgence theorem following Écalle's method by regular perturbation and majorant series which was given by M. Loday-Richaud and the author in [3].

Remark 3.2 For all $\varepsilon \in \mathcal{D}_{p}$, any of the $J$ column-blocks of $\widetilde{F}^{\varepsilon}(x)$ associated with the Jordan structure of $L$ (matrix of exponents of formal monodromy) can be positionned at the first place by means of a same permutation (hence, independent of $\varepsilon$ ) acting on the columns of $\widetilde{Y}^{\varepsilon}(x)$. Consequently, it is sufficient to prove proposition 1 in restriction to the column-block $\tilde{f}^{\varepsilon}(x)$ formed by the first $n_{1}$ (= dimension of the first Jordan block of $L$ ) columns of $\widetilde{F}^{\varepsilon}(x)$.

For all $\varepsilon \in \mathcal{D}_{p}$, the system

$$
x^{2} \frac{d Y}{d x}=A_{0}^{\varepsilon}(x) Y \quad \text { with } \quad A_{0}^{\varepsilon}(x)=\bigoplus_{j=1}^{J}\left(a_{j}^{\varepsilon} I_{n_{j}}+x L_{j}\right)
$$

has for formal fundamental solution the matrix $x^{L} e^{Q^{\varepsilon}(1 / x)}$ (recall that $L_{j}:=$ $\lambda_{j} I_{n_{j}}+J_{n_{j}}$ denotes the $j^{\text {th }}$ Jordan block of $\left.L\right)$. Thereby, according to the normalizations of $\tilde{Y}^{\varepsilon}(x)$ (cf. page 7 ), $\tilde{f}^{\varepsilon}(x)$ is uniquely determined by the first $n_{1}$ columns of the homological system

$$
x^{2} \frac{d F}{d x}=A^{\varepsilon}(x) F-F A_{0}^{\varepsilon}(x)
$$


associated with system $\left(A^{\varepsilon}\right)$ jointly with the initial condition $\tilde{f}^{\varepsilon}(0)=I_{n, n_{1}}(=$ the first $n_{1}$ columns of the identity matrix $I_{n}$ of size $n$ ) (see [1]). Therefrom, the system

$$
x^{2} \frac{d f}{d x}=A^{\varepsilon}(x) f-x f J_{n_{1}}
$$

Recall that $a_{1}^{\varepsilon}=\lambda_{1}=0$. Recall also that

$$
A^{\varepsilon}(x)=\bigoplus_{j=1}^{J}\left(a_{j}^{\varepsilon} I_{n_{j}}+x L_{j}\right)+B(x)
$$

where $B(x)$ is analytic at 0 . More precisely, splitting $B(x)=\left[B^{j ; \ell}(x)\right]$ into blocks fitting the Jordan structure of $L$, we have

$$
B^{j ; \ell}(x)=\left\{\begin{array}{ll}
O(x) & \text { if } a_{j}^{\varepsilon} \neq a_{\ell}^{\varepsilon} \\
O\left(x^{2}\right) & \text { if } a_{j}^{\varepsilon}=a_{\ell}^{\varepsilon}
\end{array} .\right.
$$

Notation 3.3 From now on, given a matrix $M$ split into blocks fitting to the Jordan structure of $L$, we denote by $M^{j ; \bullet}$ the $j$-th row-block of $M$. So, $M^{j ; \bullet}$ is a $n_{j} \times p$-matrix when $M$ is a $n \times p$-matrix.

\subsubsection{Regular perturbation}

Following J. Écalle ([2]), we consider, instead of system (3.2), the regularly perturbed system

$$
x^{2} \frac{d f}{d x}=A^{\varepsilon}(x, \alpha) f-x f J_{n_{1}}
$$

where

$$
A^{\varepsilon}(x, \alpha)=\bigoplus_{j=1}^{J}\left(a_{j}^{\varepsilon} I_{n_{j}}+x L_{j}\right)+\alpha B(x) .
$$

Like in [3], an identification of equal power in $\alpha$ shows that system (3.4) admits, for all $\varepsilon \in \mathcal{D}_{p}$, a unique formal solution of the form

$$
\tilde{f}^{\varepsilon}(x, \alpha)=\sum_{m \geq 0} \tilde{f}_{m}^{\varepsilon}(x) \alpha^{m}
$$

satisfying $\tilde{f}_{0}^{\varepsilon}(x)=I_{n, n_{1}}$ and $\tilde{f}_{m}^{\varepsilon}(x) \in M_{n, n_{1}}(\mathbb{C}[[x]])$ for all $m \geq 1$. More precisely, the components $\tilde{f}_{m}^{\varepsilon j ;}(x) \in M_{n_{j}, n_{1}}(\mathbb{C}[[x]])$ of $\tilde{f}_{m}^{\varepsilon}(x)$ are uniquely determined for all $m \geq 1$ and $j=1, \ldots, J$ as formal solutions of systems

$$
x^{2} \frac{d \tilde{f}_{m}^{\varepsilon j ; \bullet}}{d x}-a_{j}^{\varepsilon} \tilde{f}_{m}^{\varepsilon j ; \bullet}-x L_{j} \tilde{f}_{m}^{\varepsilon j ; \bullet}=B^{j ; \bullet} \tilde{f}_{m-1}^{\varepsilon}-x \widetilde{f}_{m}^{\varepsilon j ; \bullet} J_{n_{1}} .
$$


Relations (3.5) and normalizations (3.3) of $B(x)$ show in particular that

$$
\tilde{f}_{2 m-1}^{\boldsymbol{\varepsilon} j ; \bullet}(x)=O\left(x^{m}\right) \quad \text { and } \quad \tilde{f}_{2 m}^{\boldsymbol{\varepsilon} j ; \bullet}= \begin{cases}O\left(x^{m}\right) & \text { if } a_{j}^{\varepsilon}=0 \\ O\left(x^{m+1}\right) & \text { if } a_{j}^{\varepsilon} \neq 0\end{cases}
$$

for all $m \geq 1$ and $j=1, \ldots, J$.

As a result, the series $\tilde{f}^{\varepsilon}(x, \alpha)$ can be rewritten as a series in $x$ with polynomial coefficients in $\alpha$. Consequently, for all $\varepsilon \in \mathcal{D}_{p}, \widetilde{f}^{\varepsilon}(x)=\widetilde{f}^{\varepsilon}(x, 1)$ (by unicity of $\tilde{f}^{\varepsilon}(x)$ and $\left.\tilde{f}^{\varepsilon}(x, 1)\right)$ and, for all $\alpha$, the series $\tilde{f}^{\varepsilon}(x, \alpha)$ admits a formal Borel transform $\varphi^{\varepsilon}(\xi, \alpha)$ with respect to $x$ of the form

$$
\varphi^{\varepsilon}(\xi, \alpha)=\delta I_{n, n_{1}}+\sum_{m \geq 1} \varphi_{m}^{\varepsilon}(\xi) \alpha^{m}
$$

where $\varphi_{m}^{\varepsilon}(\xi) \in M_{n, n_{1}}(\mathbb{C}[[\xi]])$ denotes, for all $m \geq 1$, the Borel transform of $\tilde{f}_{m}^{\varepsilon}(x)$. In particular, the components $\varphi_{m}^{\varepsilon j ; \bullet}(\xi) \in M_{n_{j}, n_{1}}(\mathbb{C}[[\xi]])$ of $\varphi_{m}^{\varepsilon}(\xi)$ are iteratively determined for all $m \geq 1$ and $j=1, \ldots, J$ as solutions of systems

$$
\left(\xi-a_{j}^{\boldsymbol{\varepsilon}}\right) \frac{d \varphi_{m}^{\varepsilon j ; \bullet}}{d \xi}-\left(L_{j}-I_{n_{j}}\right) \varphi_{m}^{\varepsilon j ; \bullet}=\frac{d}{d \xi}\left(\widehat{B}^{j ; \bullet} * \varphi_{m-1}^{\varepsilon}\right)-\varphi_{m}^{\varepsilon j ; \bullet} J_{n_{1}} .
$$

We set $\varphi_{0}^{\varepsilon}:=\delta I_{n, n_{1}}$. Note that the Borel transforms $\widehat{B}^{j ; \bullet}$ of $B^{j ; \bullet}$ are entire functions on all $\mathbb{C}$ since $B$ is analytic at 0 . Note also that normalizations (3.3) of $B(x)$ imply that the only singularities in $\mathbb{C}$ of systems (3.6) when $\varepsilon$ runs in $\mathcal{D}_{p}$ are the Stokes values $a_{j}^{\varepsilon} \neq 0$ of $\Omega\left(\mathcal{D}_{p}\right) \subset \Omega\left(\mathcal{D}_{p}\right)$. Hence, since the domain $V$ does not meet $\Omega\left(\mathcal{D}_{p}\right) \backslash\{0\}$ and since system (3.6) depends holomorphically on the parameter $\varepsilon \in \mathcal{D}_{p}$, the following lemma:

Lemma 3.4 The function $(\xi, \varepsilon) \longmapsto \varphi_{m}^{\varepsilon}(\xi)$ is holomorphic on $V \times \mathcal{D}_{p}$ for all $m \geq 1$.

It remains to prove that the function

$$
(\xi, \varepsilon) \longmapsto \widehat{f}^{\varepsilon}(\xi)=\varphi^{\varepsilon}(\xi, 1)=\sum_{m \geq 1} \varphi_{m}^{\varepsilon}(\xi)
$$

is well-defined and holomorphic on $V \times \mathcal{D}_{p}$. This point is proved below by using a technique of majorant series satisfying a convenient system. Of course, there are many possible majorant systems. Here, we make explicit a possible one. 


\subsubsection{A convenient majorant system}

Let $\nu$ denote the minimal distance between the elements of $V$ and the elements of $\boldsymbol{\Omega}\left(\mathcal{D}_{p}\right) \backslash\{0\}$. Observe that condition (3.1) implies $\nu>0$ (see figure 4 , page 14).

We consider, for $j=1, \ldots, J$, the regularly perturbed linear system

$$
\left\{\begin{array}{l}
C_{j}\left(g^{j ; \bullet}-I_{n, n_{1}}^{j: \bullet}\right)=J_{n_{j}} g^{j ; \bullet}+g^{j ; \bullet} J_{n_{1}}-2 I_{n, n_{1}}^{j ; \bullet} J_{n_{1}}+\alpha \frac{\left|B^{j ; \bullet}\right|(x)}{x} g \\
\text { if } a_{j}=0 \\
\left(\nu-x\left|\lambda_{j}-1\right| I_{n_{j}}\right) g^{j ; \bullet}=x J_{n_{j}} g^{j ; \bullet}+x g^{j ; \bullet} J_{n_{1}}+\alpha\left|B^{j ; \bullet}\right|(x) g \\
\text { if } a_{j} \neq 0
\end{array}\right.
$$

where

- the unknown $g$ is, as previously, a $n \times n_{1}$-matrix split into row-blocks

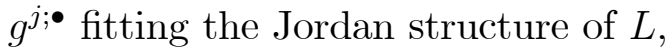

- $|B|(x)$ denotes the series $B(x)$ in which the coefficients of the powers of $x$ are replaced by their module,

- the $C_{j}$ 's are positive constants which are to be adequatly chosen below (see lemma 3).

Recall that $\lambda_{j}$ denotes the eigenvalue of the $j^{\text {th }}$ Jordan block $L_{j}$ of $L$.

Observe that the so-defined system depends on the domain $V$ but not on the parameter $\varepsilon$.

System (3.7) above has already been studied in [3] since it is actually the majorant system which has been used to prove the summable-resurgence theorem for level-one linear differential systems. In particular, it has been shown that its Borel transformed system admits, for $\alpha=1$, a solution of the form

$$
\widehat{g}(\xi)=\delta I_{n, n_{1}}+\sum_{m \geq 1} \Phi_{m}(\xi)
$$

which is entire on all $\mathbb{C}$ with exponential growth at infinity. Moreover, for any $m \geq 1, \Phi_{m}(\xi)$ belongs to $M_{n, n_{1}}\left(\mathbb{R}^{+}[[\xi]]\right)$ and is also an entire function on all $\mathbb{C}$ with exponential growth at infinity. More precisely, the components $\Phi_{m}^{j ; \bullet}(\xi) \in M_{n_{j}, n_{1}}\left(\mathbb{R}^{+}[[\xi]]\right)$ of $\Phi_{m}(\xi)$ are iteratively determined for all $m \geq 1$ and $j=1, \ldots, J$ as solutions of systems 
- Case $a_{j}=0$ :

$$
C_{j} \Phi_{m}^{j ; \bullet}=J_{n_{j}} \Phi_{m}^{j ; \bullet}+\Phi_{m}^{j ; \bullet} J_{n_{1}}+\frac{d}{d \xi}\left(\widehat{\left|B^{j ; \bullet}\right|} * \Phi_{m-1}\right) .
$$

- Case $a_{j} \neq 0$ :

$$
\nu \frac{d \Phi_{m}^{j ; \bullet}}{d \xi}=\left|\lambda_{j}-1\right| \Phi_{m}^{j ; \bullet}+J_{n_{j}} \Phi_{m}^{j ; \bullet}+\Phi_{m}^{j ; \bullet} J_{n_{1}}+\frac{d}{d \xi}\left(\widehat{\left|B^{j ; \bullet}\right|} * \Phi_{m-1}\right) .
$$

We set $\Phi_{0}:=\delta I_{n, n_{1}}$.

In addition, one can verify that all the calculations made in $[3$, section 2.5.5] to prove that system (3.7) was a convenient majorant system can also be applied in the present case. Indeed, the parameter $\varepsilon$ just acts on the Stokes values $a_{j}^{\varepsilon}$ in systems (3.6) and condition $a_{j}^{\varepsilon}=0$ (resp. $a_{j}^{\varepsilon} \neq 0$ ) is equivalent to the condition $a_{j}=0$ (resp. $a_{j} \neq 0$ ). Therefrom, the following lemma:

Lemma 3.5 (Majorant series, [3, lemma 2.9])

Let a be a positive constant such that $|\arg (\xi)| \leq$ a for all $\xi \in V$.

Let

$$
C_{j}=\frac{1-\operatorname{Re}\left(\lambda_{j}\right)}{\max _{1 \leq j \leq J} \exp \left(2 a\left|\operatorname{Im}\left(\lambda_{j}\right)\right|\right)} .
$$

Then, for all $m \geq 1, \xi \in V, \varepsilon \in \mathcal{D}_{p}$ and $j=1, \ldots, J$, the following inequalities hold:

$$
\left|\varphi_{m}^{\varepsilon j \bullet \bullet}(\xi)\right| \leq \Phi_{m}^{j ; \bullet}(|\xi|) .
$$

In particular, for all $\varepsilon \in \mathcal{D}_{p}$, the series

$$
\widehat{g}(|\xi|)=\sum_{m \geq 1} \Phi_{m}(|\xi|)
$$

is a majorant series of $\widehat{f}^{\varepsilon}(\xi)$.

Recall that lemma 3 is proved by applying Grönwall lemma to systems (3.6) defining the $\varphi_{m}^{\varepsilon j ; \bullet ' s ~ a n d ~ s y s t e m s ~ a b o v e ~ d e f i n i n g ~ t h e ~} \Phi_{m}^{j ; \bullet}$ 's.

Note that the constant $K$ given in [3, lemma 2.9] is equal to 1 in our case. Indeed, according to the definition of domain $V$, the "optimal" path $\gamma_{\xi}$ from 0 to any $\xi \in V$ used in the proof of [3, lemma 2.9] is here the straigth line $[0, \xi]$.

Before proving proposition 1, let us make some remarks about lemma 3 and calculations above. 
Remark 3.6 Like system (3.7), the function $\widehat{g}(\xi)$ depends on domain $V$ but not on the parameter $\varepsilon$.

Remark 3.7 Lemma 3 and calculations above imply the following property: there exist $c, k>0$ such that inequality

$$
\left|\widehat{f}^{\varepsilon}(\xi)\right| \leq c e^{k|\xi|}
$$

holds for all $\xi \in V$ and $\varepsilon \in \mathcal{D}_{p}$.

Remark 3.8 According to remark 4, property (3.8) can be extended to the other columns of $\widehat{F}^{\varepsilon}$ : there exist $C, K>0$ such that inequality

$$
\left|\widehat{F}^{\varepsilon}(\xi)\right| \leq C e^{K|\xi|}
$$

holds for all $\xi \in V$ and all $\varepsilon \in \mathcal{D}_{p}$.

\subsubsection{Proof of proposition 1}

We shall now prove proposition 1: lemmas 2 and 3 above tell us that the series

$$
(\xi, \varepsilon) \longmapsto \widehat{f}^{\varepsilon}(\xi)=\sum_{m \geq 1} \varphi_{m}^{\varepsilon}(\xi)
$$

is a series of holomorphic functions on $V \times \mathcal{D}_{p}$ which normally converges on all the compact sets of $V \times \mathcal{D}_{p}$. Hence, $(\xi, \varepsilon) \longmapsto \widehat{f}^{\varepsilon}(\xi)$ is well-defined and holomorphic on $V \times \mathcal{D}_{p}$ too, which achieves the proof of proposition 1 ( $c f$. remark 4).

\subsection{Dependence in $\varepsilon$ and summation}

Let us now consider an anti-Stokes direction $\theta \in \Theta$ of initial system $(A)$ and its associated sector $\Sigma_{\theta, \eta(\theta)}\left(c f\right.$. section 2.3). Recall that the set $D \Sigma_{\theta, \eta(\theta)}$ of all the directions determined by all the points of $\Sigma_{\theta, \eta(\theta)}$ are all the antiStokes directions of all systems $\left(A^{\varepsilon}\right)$ associated with $\theta$ under the action of the perturbation.

We also consider two directions $\theta+\eta / 2$ and $\theta-\eta / 2$ as in section 2.4 (cf. figure 3, page 11). Let $V^{+}$(resp. $V^{-}$) be a domain in $\mathbb{C}$ satisfying condition (3.1) above and defined by the data of an open disc centered at $0 \in \mathbb{C}$ and an open sector in $\mathbb{C}$ with vertex 0 and bisected by $\theta+\eta / 2$ (resp. $\theta-\eta / 2$ ). Note that such domains exist since $\theta \pm \eta / 2$ are singular directions for none of systems $\left(A^{\varepsilon}\right)$. 
Since $V^{+}$and $V^{-}$are domains as in section 3.1, proposition 1 and remark 7 imply the following lemma.

\section{Lemma 3.9}

1. Domain $V^{+}$

(a) For all $\xi \in V^{+}$, the function $\varepsilon \longmapsto \widehat{F}^{\varepsilon}(\xi)$ is holomorphic on $\mathcal{D}_{p}$.

(b) There exist $C^{+}, K^{+}>0$ such that inequality

$$
\left|\widehat{F}^{\varepsilon}(\xi)\right| \leq C^{+} e^{K^{+}|\xi|}
$$

holds for all $\xi \in V^{+}$and all $\varepsilon \in \mathcal{D}_{p}$.

\section{Domain $V^{-}$}

(a) For all $\xi \in V^{-}$, the function $\varepsilon \longmapsto \widehat{F}^{\varepsilon}(\xi)$ is holomorphic on $\mathcal{D}_{p}$.

(b) There exist $C^{-}, K^{-}>0$ such that inequality

$$
\left|\widehat{F}^{\varepsilon}(\xi)\right| \leq C^{-} e^{K^{-}|\xi|}
$$

holds for all $\xi \in V^{-}$and all $\varepsilon \in \mathcal{D}_{p}$.

As a result of points 1.(b) and 2.(b), the 1-sums $s_{1 ; \theta+\eta / 2}\left(\widetilde{F}^{\varepsilon}\right)$ and $s_{1 ; \theta-\eta / 2}\left(\widetilde{F}^{\varepsilon}\right)$ are respectively holomorphic, for all $\varepsilon \in \mathcal{D}_{p}$, on sectors

$$
\Sigma_{\theta+\eta / 2}\left(\frac{1}{K^{+}}\right):=\left\{x \in \mathbb{C}^{*} ;|x|<\frac{1}{K^{+}} \text {and }\left|\arg (x)-\theta-\frac{\eta}{2}\right|<\frac{\pi}{2}\right\}
$$

and

$$
\Sigma_{\theta-\eta / 2}\left(\frac{1}{K^{-}}\right):=\left\{x \in \mathbb{C}^{*} ;|x|<\frac{1}{K^{-}} \text {and }\left|\arg (x)-\theta+\frac{\eta}{2}\right|<\frac{\pi}{2}\right\}
$$

and so, according to the choice of $\eta$ ( $c f$. section 2.4), on sector

$\Sigma:=\left\{x \in \mathbb{C}^{*} ;|x|<\min \left(\frac{1}{K^{-}}, \frac{1}{K^{+}}\right)\right.$and $\left.\left(\theta-\frac{\eta}{2}\right)^{\star}<\arg (x)<\left(\theta-\frac{\eta(\theta)}{2}\right)^{\star}\right\}$.

Observe that $\Sigma$ does not depend on the parameter $\varepsilon$.

This leads us to the following result which is the central point of the proof of theorem 1 . 
Proposition 3.10 For all $x \in \Sigma$, the functions

$$
\varepsilon \longmapsto s_{1 ; \theta+\eta / 2}\left(\widetilde{F}^{\varepsilon}\right)(x) \text { and } \varepsilon \longmapsto s_{1 ; \theta-\eta / 2}\left(\widetilde{F}^{\varepsilon}\right)(x)
$$

are holomorphic on $\mathcal{D}_{p}$.

Proof. $\star$ Fix $x \in \Sigma$. For all $\varepsilon \in \mathcal{D}_{p}$, the 1 -sum $s_{1 ; \theta+\eta / 2}\left(\widetilde{F}^{\varepsilon}\right)(x)$ is given by the Borel-Laplace integral

$$
s_{1 ; \theta+\eta / 2}\left(\widetilde{F}^{\varepsilon}\right)(x)=\int_{0}^{\infty e^{i(\theta+\eta / 2)}} \widehat{F}^{\varepsilon}(\xi) e^{-\xi / x} d \xi=\int_{0}^{+\infty} \widehat{G}_{+}^{\varepsilon}(\xi) d \xi
$$

where

$$
\widehat{G}_{+}^{\varepsilon}(\xi):=\widehat{F}^{\varepsilon}\left(\xi e^{i(\theta+\eta / 2)}\right) e^{-\xi \exp (i(\theta+\eta / 2)) / x} .
$$

Since $\xi e^{i(\theta+\eta / 2)} \in V^{+}$for all $\xi \geq 0$, we can apply lemma 4 to $\widehat{G}_{+}^{\varepsilon}(\xi)$ :

- for all $\xi \geq 0$, the function $\varepsilon \longmapsto \widehat{G}_{+}^{\varepsilon}(\xi)$ is holomorphic on $\mathcal{D}_{p}$,

- for all $\xi \geq 0$ and all $\varepsilon \in \mathcal{D}_{p}$,

$$
\begin{aligned}
\left|\widehat{G}_{+}^{\varepsilon}(\xi)\right| & \leq\left|\widehat{F}^{\varepsilon}\left(\xi e^{i(\theta+\eta / 2)}\right)\right| e^{-\xi \operatorname{Re}(\exp (i(\theta+\eta / 2)) / x)} \\
& \leq C^{+} e^{-\xi\left(\operatorname{Re}(\exp (i(\theta+\eta / 2)) / x)-K^{+}\right)}:=M_{+}(\xi) .
\end{aligned}
$$

Note that $M_{+}$does not depend on $\varepsilon$. Note also that the choice " $x \in \Sigma$ " implies that $\xi \longmapsto M_{+}(\xi)$ is integrable on $[0,+\infty[$. Then, Lebesgues dominated convergence theorem applies and the function $\varepsilon \longmapsto s_{1 ; \theta+\eta / 2}\left(\widetilde{F}^{\varepsilon}\right)(x)$ is holomorphic on $\mathcal{D}_{p}$.

$\star$ The holomorphy of $\varepsilon \longmapsto s_{1 ; \theta-\eta / 2}\left(\widetilde{F}^{\varepsilon}\right)(x)$ is proved in a similar way.

\subsection{Proof of theorem 1, point 1}

Let us fix $x \in \Sigma$. Recall ( $c f$. page 11) that the perturbed Stokes matrices $\mathfrak{S}_{\theta^{\star}}^{\varepsilon}$ are uniquely determined, for all $\varepsilon \in \mathcal{D}_{p}$, by the relation

$$
Y_{\theta-\eta / 2}^{\varepsilon}(x)=Y_{\theta+\eta / 2}^{\varepsilon}(x) \mathfrak{S}_{\theta^{\star}}^{\varepsilon}
$$

where

$$
Y_{\theta \pm \eta / 2}^{\varepsilon}(x)=s_{1 ; \theta \pm \eta / 2}\left(\widetilde{F}^{\varepsilon}\right)(x) x^{L} e^{Q^{\varepsilon}(1 / x)} .
$$

Obviously, the function $\varepsilon \longmapsto Q^{\varepsilon}(1 / x)$ is holomorphic on $\mathcal{D}_{p}$. Consequently, proposition 2 above implies that the functions $\varepsilon \longmapsto Y_{\theta \pm \eta / 2}^{\varepsilon}(x)$ are also holomorphic on $\mathcal{D}_{p}$. 
On the other hand, for any $\varepsilon \in \mathcal{D}_{p}$, the matrices $Y_{\theta \pm \eta / 2}^{\varepsilon}$ are formal fundamental solutions of system $\left(A^{\varepsilon}\right)$. Thus, $Y_{\theta \pm \eta / 2}^{\varepsilon}(x) \neq 0$ for all $\varepsilon \in \mathcal{D}_{p}$ and $\varepsilon \longmapsto Y_{\theta \pm \eta / 2}^{\varepsilon}(x)^{-1}$ is still holomorphic on $\mathcal{D}_{p}$.

Theorem 1 follows since identity (2.1) implies

$$
\mathfrak{S}_{\theta^{\star}}^{\varepsilon}=Y_{\theta+\eta / 2}^{\varepsilon}(x)^{-1} Y_{\theta-\eta / 2}^{\varepsilon}(x)
$$

for all $\varepsilon \in \mathcal{D}_{p}$.

\section{References}

[1] W. Balser, W. B. Jurkat and D. A. Lutz, A general theory of invariants for meromorphic differential equations; Part I, formal invariants, Funkcialaj Ekvacioj, 22, 197-221 (1979)

[2] J. Écalle, Les fonctions résurgentes, tome III: l'équation du pont et la classification analytique des objets locaux, Publications Mathématiques d'Orsay, 85-05 (1985)

[3] M. Loday-Richaud and P. Remy, Resurgence, Stokes phenomenon and alien derivatives for level-one linear differential systems, Journal of Differential Equations, 250, 1591-1630 (2011)

[4] J.-P. Ramis, Filtration de Gevrey sur le groupe de Picard-Vessiot d'une équation différentielle irrégulière (juin 1985), in P. Deligne, B. Malgrange, J.-P. Ramis, Singularités irrégulières, Documents Mathématiques (Paris) (Mathematical Documents (Paris)), 5. Société Mathématique de France, Paris (2007)

[5] P. Remy, Stokes phenomenon for single-level linear differential systems: a perturbative approach, submitted 